\title{
Riechen und Schmecken
}

\section{Erhard Taverna}

Dr. med., Mitglied der Redaktion

Museum Tinguely Basel, Belle Haleine-Der Duft der Kunst, bis 17. Mai 2015

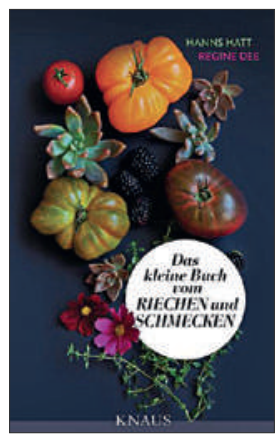

Hanns Hatt, Regine Dee

Das kleine Buch vom Riechen und Schmecken

München: Knaus Verlag; 2012. 220 Seiten. 24.90 CHF. ISBN 978-3-813-50444-6

erhard.taverna[at]saez.ch
Ein schwerer, undefinierbarer Duft füllt die Eingangshalle zum Tinguely-Museum. Pheromone, ätherische Öle, Lilienessenz, Jasmin, Eukalyptus, Gardenien und Ingwer entströmen Nebelmaschinen, Moosbeeten, Stoffsäcken, Duftpostkarten und olfaktorischen Raumskulpturen. Die Ausstellung Belle Haleine - Der Duft der Kunst* möchte dazu anregen, über vernachlässigte sensorische Fähigkeiten nachzudenken. Eine Auswahl von Kunstwerken aus den letzten zwanzig Jahren sorgt mit überraschenden Experimenten und vielen Exponaten für eine spannende Entdeckungsreise durch das Universum der Nase. Allegorische Darstellungen aus der Barockzeit und Dokumente von Künstlern der 1920er Jahre wie Marcel Duchamp, Man Ray und Carlo Carrà bilden den Auftakt zu einem ausgedehnten Rundgang, vorbei an Videoinstallationen, Sprühflaschen und mit Angstschweiss präparierten Wänden bis zum Gasgeruch in der Dunkelkammer mit dem aufgewirbelten Talkstaub im Untergeschoss. Kindern und Schwangeren nicht zu empfehlen. Verführerisches und Ekliges, Anziehendes und Abstossendes, Angst und Lust, Erinnerungen und Emotionen, alles was in der Riechbiographie des piriformen Cortex gespeichert ist, wird mit fantasievoll-ästhetischen Mitteln hervorgelockt, provoziert, bestätigt oder in Frage gestellt. Die multimediale Ausstellung erinnert daran, dass das Gedächtnis der Nase unzählige Duftmoleküle speichert, die meist unbewusst unsere Alltagshandlungen steuern. Das Reich des Riechsinns bildet den Auftakt zu weiteren geplanten Vorführungen über alle übrigen Sinne. Auch Krankheiten haben einen Geruch. Seit bekannt ist, dass Hunde Krebs riechen können, arbeiten Forscher an Analysemethoden zur Diagnostik. Dem Atemtest zum Nachweis von Helicobacter pylori könnten bald chemische Sensoren zur Erkennung typischer Krankheitssignaturen folgen. Vieles spricht für individuelle Atemmuster, die sich mit geeigneten Analysegeräten und regelmässigen Proben auch als Frühwarnsystem für gesunde Personen eignen würden. Von der Nase lernen lautet die Devise. Doch dazu braucht es Forscher, wie Hanns Hatt, Leiter des Lehrstuhls für Zellphysiologie an der Universität Bochum. Das menschliche Duft-Alphabet hat 350 Buchstaben. Rund 20 dieser Riechrezeptoren der menschlichen Nase hat sein Team isoliert, von denen wiederum jede Zilie der dreissig Millionen Riechsinneszellen nur eine Sorte besitzt. Das Duftmolekül passt zum Rezeptor wie der Schlüssel zum Schloss, wobei dieses die Botschaft nicht nur lesen, sondern auch vervielfältigen kann. Längst sind Pheromone nicht nur eine Domäne der Insektenforscher. Androstenon und Androstenol sorgen für gute Stimmung, Angstschweiss, Muttermilch und Kopuline aus dem Vaginalsekret beflügeln, wie Ambra, Moschus und Zibet, die Parfumindustrie. Wen wundert es da, dass auch das Museum Tinguely am Valentinstag zur ersten Schweizer Pheromonparty eingeladen hat. 2005 konnte das Bochumer Team weltweit zum ersten Mal nachweisen, dass Riechrezeptoren aus der Nase in vielen Organen unseres Körpers vorkommen. Für Schlagzeilen sorgte die Entdeckung, dass Spermien mit Riechrezeptoren ausgestattet sind, die sich nach dem Maiglöckchenduft der Eizelle orientieren. In der Prostata finden sich die Veilchenrezeptoren aus der Nase. Prostatakrebszellen im Reagenzglas stellen in Gegenwart des Veilchendufts ihr Wachstum ein. Duftmoleküle wirken als chemische Signalstoffe in Haut, Leber und im Darm.

Gerüche scheinen weit mehr als Libido und Abneigung zu steuern. Wie sie zusammen mit den Geschmacksknospen der Zunge und den sensiblen Trigeminusnerven das limbische System zum Zentrum der Genüsse machen, hat der in Zoologie, Humanphysiologie und Medizin promovierte Hanns Hatt mit der Journalistin Regine Dee anschaulich beschrieben. «Das kleine Buch vom Riechen und Schmecken» ist ein vergnügliches Kompendium zum heutigen Wissen über Sex, Kulinarik und vieles mehr. Ansprechend gestaltet, informativ, humorvoll und gut verständlich, inklusive Nasentraining und Gehirnjogging, mit Riechspielen für Kinder und einer Anleitung für Weinkenner. Dass auch die Augen Duft und Aroma mitgestalten, zeigen die vielen Versuchsanordnungen beim Degustieren oder bei der Blindverkostung von Sommeliers. Doch aller Raffinesse zum Trotz, auch unverfälschte Körpergerüche haben ihren Auftritt. «Wasche dich nicht, ich komme», lautete die legendäre Botschaft Napoleons an Joséphine, wenn er von einem Feldzug zurückkehrte. 